J. Math. Phys., Vol 39, 1998 Report No: UWThPh-1997-38 ESI-Preprint No: 505

\title{
Geodesics and geodesic deviation for impulsive gravitational waves
}

\author{
R. Steinbauer \\ Institute for Theoretical Physics, University of Vienna \\ Boltzmanng. 5, A-1090 Wien, Austria \\ E-mail: stein@doppler.thp.univie.ac.at
}

(Received 4 November 1997; accepted for publication 19 November 1997)

The geometry of impulsive pp-waves is explored via the analysis of the geodesic and geodesic deviation equation using the distributional form of the metric. The geodesic equation involves formally ill-defined products of distributions due to the nonlinearity of the equations and the presence of the Dirac $\delta$-distribution in the space time metric. Thus, strictly speaking, it cannot be treated within Schwartz's linear theory of distributions. To cope with this difficulty we proceed by first regularizing the $\delta$-singularity, then solving the regularized equation within classical smooth functions and, finally, obtaining a distributional limit as solution to the original problem. Furthermore it is shown that this limit is independent of the regularization without requiring any additional condition, thereby confirming earlier results in a mathematical rigorous fashion. We also treat the Jacobi equation which, despite being linear in the deviation vector field, involves even more delicate singular expressions, like the "square" of the Dirac $\delta$-distribution. Again the same regularization procedure provides us with a perfectly well behaved smooth regularization and a regularization-independent distributional limit. Hence it is concluded that the geometry of impulsive pp-waves can be described consistently using distributions as long as careful regularization procedures are used to handle the ill-defined products. PACS-numbers: 04.20.Cv, 04.20.-q, 02.20.Hq, 04.30.-w

\section{INTRODUCTION}

Plane fronted gravitational waves with parallel rays (pp-waves) are spacetimes characterized by the existence of a covariantly constant null vector field, which can be used to write the metric tensor in the form 1

$$
d s^{2}=H(u, x, y) d u^{2}-d u d v+d x^{2}+d y^{2},
$$

where $u, v$ is a pair of null coordinates $(u=t-z, v=t+z)$ and $x, y$ are transverse (Cartesian) coordinates. In this paper we shall deal especially with impulsive (in the diction of Penrose2) pp-waves where the profile function $H$ is proportional to a $\delta$-distribution, i.e., takes the form $H(u, x, y)=f(x, y) \delta(u)$, where we leave the (smooth) function $f$ of the transverse coordinates arbitrary for the moment. This metric is flat everywhere exept on the null hypersurface $u=0$, where it has a $\delta$-shaped "shock".

Such spacetimes arise most prominently as ultrarelativistic limits of black hole geometries as first derived by Aichelburg and Sexl for the Schwarzschild case ${ }^{3}$. On the other hand Penrose has given a more intrinsic description of such spacetimes by 
his "scissor and paste" approach 1 , which essentially consists of glueing together two pieces of Minkowski spacetime along the null hypersurface $u=0$ with a shift in the $v$-direction. A similar idea was used by Dray and t'Hooft, who introduced a coordinate shift along geodesics in Minkowski space time to rederive the Aichelburg-Sexl geometry as well as Penrose's junction conditions from the field equations.

The philosophy of the present work is somehow complementary. We take the $\delta$ shaped metric literally and try to explore the properties of this geometry via investigation of geodesics and the geodesic deviation, thereby following the approaches of Ferrari, Pendenza and Veneziand 8 and Balasine.

The main purpose of this work is to deal with the singular, i.e., distributional quantities in a mathematically rigorous fashion. A thorough analysis of the geodesic equation shows that it involves ill-defined products of the $\delta$-distribution with the step function. This difficulty can be circumvented by a proper regularization procedure providing us with a perfectly well behaved smoothapproximation, which has a distributional limit coinciding with the earlier results 9.6 . However, we neither have to impose "multiplication rules" like $\delta \theta=(1 / 2) \delta$, nor to use any additional requirements as the constancy of the norm of the geodesic's tangent vector across the shock. In fact, this property comes out as a result in our approach. The details of this calculation are given in Sec. II.

Further investigations show that the Jacobi equation involves ill-defined terms of an even worse type, such as the "square" of the Dirac $\delta$-distribution and the square of the step function times the $\delta$-distribution. However, our regularization strategy again provides us with a smooth approximation and a well behaved distributional limit, even in this case, where it seems to be hopeless to use "ad-hoc extensions" of Schwartz's linear distribution theory such as special "multiplication rules". We give the details of the rather lengthy calculation in Sec. III. Our distributional "solution" of the Jacobi equation fits perfectly well in the (heuristically) expected picture, showing the consistency of our approach. Finally, we make some comments and give an outlook to future work in Sec. IV.

\section{GEODESIC EQUATION}

We start with an impulsive pp-wave metric of the form

$$
d s^{2}=f\left(x^{i}\right) \delta(u) d u^{2}-d u d v+\left(d x^{i}\right)^{2},
$$

where $x^{i}(i=1,2)$ denote the transverse coordinates. It is straightforward to derive the geodesic equation. The nonvanishing Christoffel symbols are

$$
\Gamma_{u u}^{v}=-f \dot{\delta}, \quad \Gamma_{u u}^{i}=-\frac{1}{2} \partial_{i} f \delta, \quad \Gamma_{u i}^{v}=\Gamma_{i u}^{v}=-\partial_{i} f \delta,
$$

where we have denoted the partial derivatives of $f$ by $\partial_{i} f$ and the derivative of the $\delta$-distribution by $\dot{\delta}$. Hence we get the equations

$$
\begin{aligned}
u^{\prime \prime} & =0, \\
v^{\prime \prime} & =f \dot{\delta}+2\left(\partial_{i} f x^{i \prime}\right) u^{\prime} \delta, \\
x^{i \prime \prime} & =\frac{1}{2} \partial_{i} f u^{\prime 2} \delta,
\end{aligned}
$$

where ' denotes the derivative with respect to an affine parameter and summation over $i$ is understood. We use the first equation to introduce $u$ as a new affine parameter (there excluding trivial geodesics parallel to the shock hypersurface) to get 


$$
\begin{aligned}
\ddot{v}(u) & =f\left(x^{i}(u)\right) \dot{\delta}(u)+2\left(\partial_{i} f\left(x^{i}(u)\right) \dot{x}^{i}(u)\right) \delta(u), \\
\ddot{x}^{i}(u) & =\frac{1}{2} \partial_{i} f\left(x^{i}(u)\right) \delta(u),
\end{aligned}
$$

where again denotes the derivative with respect to $u$ and we have inserted all the dependences explicitly. Equations (5) form a system of three coupled, nonlinear ODEs of second order in the vectorspace $\mathcal{D}^{\prime}$ of distributions. For $u \neq 0$ all the right hand sides vanish, which is clear from the form of the metric tensor, and we expect the geodesics to be broken, possibly refracted straight lines. However, if we take a closer look at system (5) we see immediately that the first equation cannot be taken literally in the sense of distributions as the terms $\dot{x}^{i} \delta$ involve ill-defined products of the $\delta$-distribution with the step function.

To analyze the situation in some more detail we integrate the last two equations using the (distributional) identity $f\left(x^{i}(u)\right) \delta(u)=f\left(x^{i}(0)\right) \delta(u)$ to get

$$
x^{i}(u)=\text { initial values }+\frac{1}{2} \partial_{i} f\left(x^{i}(0)\right) u_{+},
$$

where we have denoted the "kink"-function $u \theta(u)$ by $u_{+}$. Note, however, that distributions can only be multiplied by $C^{\infty}$-functions, whereas it's not clear a priori that the solution $x^{i}(u)$ will be smooth; in fact as suggested by equation (6) and as shown later in our calculations the solution will not even be differentiable at $u=0$. If we still try to go on by brute force it comes even worse: inserting (6) into the first equation (5) we see that the term $\dot{x}^{i}(u) \delta(u)$ gives rise to the ill-defined product $\theta \delta$. (For some further comments on this product see 2 , p 21.)

To overcome the undefinedness described in detail above, we apply a careful regularization procedure. More precisely we regularize the $\delta$-distribution by a (standard) mollifier or model $\delta$-net, i.e., a net $\rho_{\epsilon}$, defined as follows. Let $\rho$ be a smooth function with support contained in the interval $[-1,1]$ and $\int \rho=1$; now put $\rho_{\epsilon}(x)=(1 / \epsilon) \rho(x / \epsilon),(\epsilon>0)$. Hence system (5) takes the regularized form

$$
\begin{aligned}
& \ddot{v}_{\epsilon}(u)=f\left(x_{\epsilon}^{i}(u)\right) \dot{\rho}_{\epsilon}(u)+2 \partial_{i} f\left(x_{\epsilon}^{i}(u)\right) \dot{x}_{\epsilon}^{i}(u) \rho_{\epsilon}(u), \\
& \ddot{x}_{\epsilon}^{i}(u)=\frac{1}{2} \partial_{i} f\left(x_{\epsilon}^{i}(u)\right) \rho_{\epsilon}(u) .
\end{aligned}
$$

Next we choose initial conditions in $u=-1$ (i.e. "long before the shock"), more precisely

$$
\begin{array}{ll}
v_{\epsilon}(-1)=v_{0}, & x_{\epsilon}^{i}(-1)=x_{0}^{i}, \\
\dot{v}_{\epsilon}(-1)=\dot{v}_{0}, & \dot{x}_{\epsilon}^{i}(-1)=\dot{x}_{0}^{i},
\end{array}
$$

for all $\epsilon$. Note that choosing initial conditions in $u=0$ would mean to "start at the shock" and one cannot expect to (and indeed does) end up with a regularization independent result in this case.

Due to the regularization procedure we now have to deal with the fully nonlinear character of the last equation of (7). However, as shown in appendix IV the special form of the right hand side of the equation guarantees global existence of the solutions for small $\epsilon$. These are given implicitly by

$$
\begin{aligned}
& v_{\epsilon}(u)=v_{0}+\dot{v}_{0}(1+u)+\left(\theta * \theta * \ddot{v}_{\epsilon}\right)(u), \\
& x_{\epsilon}^{i}(u)=x_{0}^{i}+\dot{x}_{0}^{i}(1+u)+\left(\theta * \theta * \ddot{x}_{\epsilon}^{i}\right)(u),
\end{aligned}
$$

where "*" denotes convolution. 
We are going to calculate the (distributional) limits of the solutions (9) as $\epsilon$ tends to zero. Since distributions supported in an acute cone form a convolution algebra (where, in particular, convolution is a separately countinuous operation) it suffices to calculate the limits of the right hand sides of (7); the distributional limits of the solutions (9) are then computed simply by integration.

We begin with the latter two equations of system (7) and choose a test function $\varphi$. We have to calculate the limit of

$$
\frac{1}{2} \int_{\mathbb{R}} \partial_{i} f\left(x_{\epsilon}^{i}(u)\right) \rho_{\epsilon}(u) \varphi(u) d u=\frac{1}{2} \int_{-1}^{1} \partial_{i} f\left(x_{\epsilon}^{i}(\epsilon u)\right) \rho(u) \varphi(\epsilon u) d u
$$

Since (for small $\epsilon$ ) $x_{\epsilon}(u)$ is bounded uniformly on compact sets (see Appendix IV] we can use Lebesgue's dominated convergence. Hence the only term we have to compute is

$$
\begin{aligned}
\lim _{\epsilon \rightarrow 0} x_{\epsilon}^{i}(\epsilon u) & =\lim \left(x_{0}^{i}+\dot{x}_{0}^{i}(1+\epsilon u)+\frac{1}{2} \int_{-\epsilon}^{\epsilon u} \int_{-\epsilon}^{v} \partial_{i} f\left(x_{\epsilon}^{i}(s)\right) \rho_{\epsilon}(s) d s d v\right) \\
& =x_{0}^{i}+\dot{x}_{0}^{i}+\frac{1}{2} \lim \underbrace{\int_{-\epsilon u}^{\epsilon} \int_{-1}^{v / \epsilon} \partial_{i} f\left(x_{\epsilon}^{i}(\epsilon s)\right) \rho(s) d s d v}_{\left|\iint\right| \leq\left\|\partial_{i} f\right\|\|\rho\| \iint d s d v} .
\end{aligned}
$$

For $\epsilon \rightarrow 0$ the last integral gives zero, since in the limit the range of integration only covers a set of zero measure. Hence we get (as expected)

$$
\ddot{x}_{\epsilon}^{i}(u)=\frac{1}{2} \partial_{i} f\left(x_{\epsilon}^{i}(u)\right) \rho_{\epsilon}(u) \rightarrow \frac{1}{2} \partial_{i} f\left(x_{0}^{i}+\dot{x}_{0}^{i}\right) \delta(u),
$$

within distributions.

Next we turn to the "critical" first equation in (7) and again calculate the limit of the right hand side, i.e., of the expression

$$
\begin{aligned}
\ddot{v}_{\epsilon}(u) & =f\left(x_{\epsilon}^{i}(u)\right) \dot{\rho}_{\epsilon}(u)+2 \partial_{i} f\left(x_{\epsilon}^{i}(u)\right) \dot{x}_{\epsilon}^{i}(u) \rho_{\epsilon}(u) \\
& =\left(f\left(x_{\epsilon}^{i}(u)\right) \rho_{\epsilon}(u)\right)^{\cdot}+\partial_{i} f\left(x_{\epsilon}^{i}(u)\right) \dot{x}_{\epsilon}^{i}(u) \rho_{\epsilon}(u) .
\end{aligned}
$$

The limit of the first term is easily seen to be $f\left(x_{0}^{i}+\dot{x}_{0}^{i}\right) \dot{\delta}(u)$, and we are only left with the second term which contains the "critical products". Inserting (9) and (7) respectively, we have

$$
\begin{aligned}
\partial_{i} f\left(x_{\epsilon}^{i}\right) \dot{x}_{\epsilon}^{i} \rho_{\epsilon} & =\partial_{i} f\left(x_{\epsilon}^{i}\right)\left(\dot{x}_{0}^{i}+\theta * \ddot{x}_{\epsilon}^{i}\right) \rho_{\epsilon} \\
& =\partial_{i} f\left(x_{\epsilon}^{i}\right) \dot{x}_{0}^{i} \rho_{\epsilon}+\partial_{i} f\left(x_{\epsilon}^{i}\right)\left(\theta *\left[\frac{1}{2} \partial_{i} f\left(x_{\epsilon}^{i}\right) \rho_{\epsilon}\right]\right) \rho_{\epsilon}
\end{aligned}
$$

The limit of the first term again is easily seen to give $\dot{x}_{0}^{i} \partial_{i} f\left(x_{0}^{i}+\dot{x}_{0}^{i}\right) \delta(u)$, and we are finally left with the task of computing the limit of the convolution term, i.e., the expression " $\theta \delta$ ". Using

$$
\left(\theta *\left[\partial_{i} f\left(x_{\epsilon}^{i}\right) \rho_{\epsilon}\right]\right)(u)=\int_{-\infty}^{u} \partial_{i} f\left(x_{\epsilon}^{i}(s)\right) \rho_{\epsilon}(s) d s=\int_{-1}^{u / \epsilon} \partial_{i} f\left(x_{\epsilon}^{i}(\epsilon s)\right) \rho(s) d s
$$


and again denoting by $\varphi$ a test function we get

$$
\begin{aligned}
\lim _{\epsilon \rightarrow 0}( & \left.\frac{1}{2} \int_{-1}^{1} \partial_{i} f\left(x_{\epsilon}^{i}(\epsilon u)\right)\left[\int_{-1}^{u} \partial_{i} f\left(x_{\epsilon}^{i}(\epsilon s)\right) \rho(s) d s\right] \rho(u) \varphi(\epsilon u) d u\right) \\
= & \frac{1}{2}\left(\partial_{i} f\left(x_{0}^{i}+\dot{x}_{0}^{i}\right)\right)^{2} \varphi(0) \underbrace{\int_{-1}^{1}\left[\int_{-1}^{u} \rho(s) d s\right] \rho(u) d u}_{1 / 2} \\
= & \frac{1}{4}\left(\partial_{i} f\left(x_{0}^{i}+\dot{x}_{0}^{i}\right)\right)^{2} \varphi(0) .
\end{aligned}
$$

Collecting things together we have

$$
\lim _{\epsilon \rightarrow 0} \ddot{v}_{\epsilon}=f\left(x_{0}^{i}+\dot{x}_{0}^{i}\right) \dot{\delta}+\dot{x}_{0}^{i} \partial_{i} f\left(x_{0}^{i}+\dot{x}_{0}^{i}\right) \delta+\frac{1}{4}\left(\partial_{i} f\left(x_{0}^{i}+\dot{x}_{0}^{i}\right)\right)^{2} \delta
$$

within distributions.

At the end of this section let us give a summary on what we have done so far. We have regularized the distributionally ill-defined geodesic equations (5) by replacing the $\delta$-distribution by a (generic) class of mollifiers to obtain (7). These equations, although nonlinear, provide us with global solutions $v_{\epsilon}$ and $x_{\epsilon}^{i}$ for small $\epsilon$ (as shown in appendix [V], implicitly given by (9). Using the latter formula we have shown that the smooth solutions have a regularization independent distributional limit given by

$$
\begin{aligned}
& v_{\epsilon}(u) \rightarrow v_{0}+\dot{v}_{0}(1+u)+f(0) \theta(u)+\partial_{i} f(0)\left(\dot{x}_{0}^{i}+\frac{1}{4} \partial_{i} f(0)\right) u_{+}, \\
& x_{\epsilon}^{i}(u) \rightarrow x_{0}^{i}+\dot{x}_{0}^{i}(1+u)+\frac{1}{2} \partial_{i} f(0) u_{+},
\end{aligned}
$$

where we have used the abbreviation $f(0)=f\left(x_{0}^{i}+\dot{x}_{0}^{i}\right)$.

Hence viewed distributionally the geodesics are given by refracted, broken straight lines as expected. Of course equations (18) coincide with the earlier results 1 , 2 . However from the point of view of our approach the (deeper) reason why here the "rule" $\theta \delta=(1 / 2) \delta$ used by 8 (which in fact coincides with the "determination of the point value" $\theta(0)=1 / 2$ used by 9 ) leads to a physically reasonable result is the following: The geodesic equations involve only one singular object and hence the $\delta$ 's as well as the $\theta$ 's appearing above share the same root: namely the $\delta$-shaped wave profile. Hence, when regularizing the equations (which in fact corresponds to the physical idea of viewing the impulsive wave as an idealized sandwich wave) both factors of the ill-defined product naturally involve the same regularization which immediately leads to the (regularization-independent) result $\rho_{\epsilon} \int \rho_{\epsilon} \rightarrow(1 / 2) \delta$.

We thus conclude that the geodesic equation can be treated consistently by regularization, leading to a regularization-independent distributional result. This, of course, is only possible due to the relatively mild character of the singular terms which allows for a distributional limit of the solutions to the regularized problem at all. However, we shall see in a moment that even in the considerably more complicated case of the Jacobi equation our strategy can be applied successfully. 


\section{JACOBI EQUATION}

In this section we solve the Jacobi equation for an impulsive pp-wave. To keep formulas more transparent we restrict ourselves to the axisymmetric case. More precisely we restrict the function $f$ of the transverse coordinates $x^{i}=(x, y)$ in the metric tensor (2) to depend on the two-radius $r=\sqrt{x^{2}+y^{2}}$ only and work entirely within the $y=0$-hypersurface (initial conditions $x_{0}^{2} \equiv y_{0}=0=\dot{x}_{0}^{2} \equiv \dot{y}_{0}$ ). Furthermore we take initial values $v_{0}=0=\dot{x}_{0}$. With these assumptions equations (18) simplify to

$$
\begin{aligned}
v_{\epsilon}(u) & \rightarrow \dot{v}_{0}(1+u)+f\left(x_{0}\right) \theta(u)+\frac{1}{4} f^{\prime}\left(x_{0}\right)^{2} u_{+}, \\
x_{\epsilon}^{1}(u) \equiv x_{\epsilon}(u) & \rightarrow x_{0}+\frac{1}{2} f^{\prime}\left(x_{0}\right) u_{+}, \\
x_{\epsilon}^{2}(u) \equiv y_{\epsilon}(u) & =0,
\end{aligned}
$$

where $f^{\prime}$ denotes the derivative with respect to the single variable $r=x$. Hence we shall deal with a geodesic tangent vector of the form

$$
T^{a}(u)=\left(\begin{array}{l}
1 \\
\dot{v}_{0}+f\left(x_{0}\right) \delta(u)+\frac{1}{4} f^{\prime}\left(x_{0}\right)^{2} \theta(u) \\
\frac{1}{2} f^{\prime}\left(x_{0}\right) \theta(u) \\
0
\end{array}\right)
$$

where we are going to use the abbreviations $A:=f\left(x_{0}\right) \delta(u)+(1 / 4) f^{\prime}\left(x_{0}\right)^{2} \theta(u)$ and $B:=(1 / 2) f^{\prime}\left(x_{0}\right) \theta(u)$ for its components.

Our next task is to compute the explicit form of the Jacobi equation $\frac{D^{2} N^{a}}{d u^{2}}=$ $-R_{b c d}^{a} T^{b} T^{d} N^{c}$ for a vector field $N^{a}(u)=\left(N^{u}(u), N^{v}(u), N^{x}(u), N^{y}(u)\right)$ over the geodesic. After some (tedious) calculations we end up with the following form of the system

$$
\begin{aligned}
\ddot{N}^{u} & =0, \\
\ddot{N}^{v} & =2\left[N^{x} f^{\prime} \delta\right]^{\cdot}-N^{x} f^{\prime} \dot{\delta}+\left[N^{u} f \delta\right]^{*}-N^{u} f^{\prime \prime} B^{2} \delta-N^{u} f^{\prime} \dot{B} \delta, \\
\ddot{N}^{x} & =\left[\dot{N}^{u} f^{\prime}+\frac{1}{2} N^{x} f^{\prime \prime}\right] \delta+\frac{1}{2} f^{\prime} N^{u} \dot{\delta}, \\
\ddot{N}^{y} & =0,
\end{aligned}
$$

where we have suppressed the dependence on the parameter $u$ and the variable $x$. Equations (21) form a system of four coupled ODE's linear in the components of the vector field $N^{a}$ but nonlinear in the derivatives of the metric. From the fact that $B$ involves the step function we see immediately that (in the second equation) we again have to deal with distributionally ill-defined expressions, but now of even worse type than before. Indeed the term $\dot{B} \delta$ is proportional to the "square" of the Dirac $\delta$-distribution, and the term $B^{2} \delta$ involves an expression " $\theta^{2} \delta$ ". Note, however, that the critical terms arise from the second covariant derivative, where some of the Christoffel symbols get multiplied, and not from the Riemann tensor which components are just proportional to the $\delta$-distribution. To overcome these problems we apply the same regularization procedure as in the case of the geodesic equation. In particular, we use the regularized geodesic tangent vector

$$
T_{\epsilon}^{a}=\left(\begin{array}{l}
1 \\
\dot{v}_{0}+\theta * \ddot{v}_{\epsilon} \\
\theta * \ddot{x}_{\epsilon} \\
0
\end{array}\right)
$$


where

$\ddot{v}_{\epsilon}(u)=f\left(x_{\epsilon}(u)\right) \dot{\rho}_{\epsilon}(u)+2 f^{\prime}\left(x_{\epsilon}(u)\right) \dot{x}_{\epsilon}(u) \rho_{\epsilon}(u), \quad \ddot{x}_{\epsilon}(u)=(1 / 2) f^{\prime}\left(x_{\epsilon}(u)\right) \rho_{\epsilon}(u)$ and from now on we use the new abbreviations $A_{\epsilon}:=\theta * \ddot{v}_{\epsilon}$ and $B_{\epsilon}:=\theta * \ddot{x}_{\epsilon}$. Denoting the regularized Jacobi field by $N_{\epsilon}^{a}(u)=\left(N_{\epsilon}^{u}(u), N_{\epsilon}^{v}(u), N_{\epsilon}^{x}(u), N_{\epsilon}^{y}(u)\right)$, system (21) takes the regularized form

$$
\begin{aligned}
\ddot{N}_{\epsilon}^{u}= & 0, \\
\ddot{N}_{\epsilon}^{v}= & 2\left[N_{\epsilon}^{x} f^{\prime}\left(x_{\epsilon}\right) \rho_{\epsilon}\right] \cdot-N_{\epsilon}^{x} f^{\prime}\left(x_{\epsilon}\right) \dot{\rho}_{\epsilon}+\left[N_{\epsilon}^{u} f\left(x_{\epsilon}\right) \rho_{\epsilon}\right]^{.}- \\
& N_{\epsilon}^{u} f^{\prime \prime}\left(x_{\epsilon}\right)\left[\theta * \ddot{x}_{\epsilon}\right]^{2} \rho_{\epsilon}-N_{\epsilon}^{u} f^{\prime}\left(x_{\epsilon}\right) \ddot{x}_{\epsilon} \rho_{\epsilon}, \\
\ddot{N}_{\epsilon}^{x}= & {\left[\dot{N}_{\epsilon}^{u} f^{\prime}\left(x_{\epsilon}\right)+\frac{1}{2} N_{\epsilon}^{x} f^{\prime \prime}\left(x_{\epsilon}\right)\right] \rho_{\epsilon}+\frac{1}{2} f^{\prime}\left(x_{\epsilon}\right) N_{\epsilon}^{u} \dot{\rho}_{\epsilon}, } \\
\ddot{N}_{\epsilon}^{y}= & 0 .
\end{aligned}
$$

To maintain transparency of formulae we choose appropriate and simple initial conditions on the Jacobi field $N_{\epsilon}^{a}$ at $u=-1$, i.e.,

$$
\begin{aligned}
& N_{\epsilon}^{a}(-1)=(0,0,0,0), \\
& \dot{N}_{\epsilon}^{a}(-1)=(a, b, 0,0),
\end{aligned}
$$

for all $\epsilon$. Note that this corresponds to a focal point at $u=-1$, and that "nearby" geodesics have relative initial velocities only in the $u$ - and $v$-direction.

The first and the last equation of system (23) are easily solved to give

$$
\begin{aligned}
& N_{\epsilon}^{u}(u)=a(1+u), \\
& N_{\epsilon}^{y}(u)=0 .
\end{aligned}
$$

For the remaining, more complicated equations of system (23) we apply the same strategy as in the preceeding section. Since the equations are linear in the components of the deviation vector field we obtain globally defined (smooth) solutions which, due to the initial conditions (24), are implicitly given by

$$
\begin{aligned}
& N_{\epsilon}^{v}(u)=b(1+u)+\left[\theta * \theta * \ddot{N}_{\epsilon}^{v}\right](u), \\
& N_{\epsilon}^{x}(u)=\left[\theta * \theta * \ddot{N}_{\epsilon}^{x}\right](u) .
\end{aligned}
$$

Again it suffices to compute the distributional limits of the right hand sides of equation (23), since by continuity of the convolution we immediately get the limits of $N_{\epsilon}^{v}$ and $N_{\epsilon}^{x}$.

We start with the third equation of the system (23). The main problem we have to face here is due to the fact that the unknown function $N_{\epsilon}^{x}$ (which, in the limit, we cannot even expect to be continuous at $u=0$ ) appears on the right hand side. Inserting the initial conditions (24) and the solutions (25) we get

$$
\ddot{N}_{\epsilon}^{x}(u)=\underbrace{a f^{\prime}\left(x_{\epsilon}(u)\right) \rho_{\epsilon}(u)}_{I_{\epsilon}}+\underbrace{\frac{1}{2} f^{\prime}\left(x_{\epsilon}(u)\right) a(1+u) \dot{\rho}_{\epsilon}(u)}_{I I_{\epsilon}}+\underbrace{\frac{1}{2} N_{\epsilon}^{x}(u) f^{\prime \prime}\left(x_{\epsilon}(u)\right) \rho_{\epsilon}(u)}_{I I I_{\epsilon}} .
$$

The distributional limits of the first two terms in equation $(27)$ are easily seen (using similar methods as in the previous section) to be 


$$
\begin{aligned}
\lim _{\epsilon \rightarrow 0} I_{\epsilon} & =a f^{\prime}\left(x_{0}\right) \delta, \\
\lim _{\epsilon \rightarrow 0} I I_{\epsilon} & =\frac{1}{2} a f^{\prime}\left(x_{0}\right)(\dot{\delta}-\delta)-\frac{1}{8} a f^{\prime}\left(x_{0}\right) f^{\prime \prime}\left(x_{0}\right) \delta .
\end{aligned}
$$

However, the last term in equation (27) requires a more detailed analysis. Inserting again $\theta * \theta * \ddot{N}_{\epsilon}^{x}$ for the unknown function $N_{\epsilon}^{x}$ and denoting by $\varphi$ a test function we obtain

$$
\begin{aligned}
& I I I_{\epsilon}(u)= \\
& \frac{1}{2} \epsilon \int_{-1}^{1} \varphi(\epsilon u) f^{\prime \prime}\left(x_{\epsilon}(\epsilon u)\right) \rho(u) \int_{-1}^{u} \int_{-1}^{s}\left[a f^{\prime}\left(x_{\epsilon}(\epsilon r)\right)+\frac{1}{2} N_{\epsilon}^{x}(\epsilon r) f^{\prime \prime}\left(x_{\epsilon}(\epsilon r)\right)\right] \rho(r) d r d s d u \\
& +\frac{1}{4} a \int_{-1}^{1} \varphi(\epsilon u) f^{\prime \prime}\left(x_{\epsilon}(\epsilon u)\right) \rho(u) \int_{-1}^{u} \int_{-1}^{s} f^{\prime}\left(x_{\epsilon}(\epsilon r)\right) \dot{\rho}(r) d r d s d u \\
& +\frac{1}{4} a \epsilon \int_{-1}^{u} \varphi(\epsilon u) f^{\prime \prime}\left(x_{\epsilon}(\epsilon u)\right) \rho(u) \int_{-1}^{s} \int_{-1}^{s} f^{\prime}\left(x_{\epsilon}(\epsilon r)\right) r \dot{\rho}(r) d r d s d u .
\end{aligned}
$$

The last term in equation (29) goes to zero, since $\varphi, f^{\prime}, f^{\prime \prime}$ are smooth functions and $x_{\epsilon}$ is bounded uniformly on compact sets (see appendix IV). For the same reasons the second summand in equation (29) approaches the limit

$$
\frac{1}{8} a \varphi(0) f^{\prime}\left(x_{0}\right) f^{\prime \prime}\left(x_{0}\right),
$$

whereas the first line also vanishes in the limit, since $N_{\epsilon}^{x}(\epsilon r)$ is bounded on the compact range of integration. (Note, however, that $\lim N_{\epsilon}^{x}$ is not continuous at 0 !) In some more detail the latter argument can be seen from

$$
\begin{aligned}
N_{\epsilon}^{x}(\epsilon u)= & \int_{-1}^{u} \int_{-1}^{s}\left\{a f^{\prime}\left(x_{\epsilon}(\epsilon r)\right) \epsilon \rho(r)+\frac{1}{2} a f^{\prime}\left(x_{\epsilon}(\epsilon r)\right)[\dot{\rho}(r)+r \epsilon \dot{\rho}(r)]\right\} d r d s \\
& +\frac{1}{2} \int_{-\epsilon}^{\epsilon u} \int_{-1}^{s / \epsilon} N_{\epsilon}^{x}(\epsilon r) f^{\prime \prime}\left(x_{\epsilon}(\epsilon r)\right) \rho(r) d r d s
\end{aligned}
$$

where we have now used equation (26) for the third time. Hence we have for $u \leq T$, where $T$ is some constant, and for fixed $\epsilon$

$$
\begin{aligned}
\left|N_{\epsilon}^{x}(\epsilon u)\right| & \leq C+C^{\prime} \int_{-\epsilon}^{\epsilon u} \int_{-1}^{s / \epsilon}\left|N_{\epsilon}^{x}(\epsilon r)\right||\rho(r)| d r d s \\
& \leq C+2 C^{\prime} \int_{-1}^{u}\left|N_{\epsilon}^{x}(\epsilon r)\right||\rho(r)| d r
\end{aligned}
$$

where $C=C(T)$ and $C^{\prime}$ denote constants. From Gronwall's inequality we gain

$$
\left|N_{\epsilon}^{x}(\epsilon u)\right| \leq C e^{2 C^{\prime} \int_{-1}^{u}|\rho(r)| d r},
$$

which is bounded by a constant for $u \leq T$. 
Collecting things together (equations (28) and (30) ) we find that within distributions

$$
\ddot{N}_{\epsilon}^{x} \rightarrow \frac{1}{2} a f^{\prime}\left(x_{0}\right)(\delta+\dot{\delta}),
$$

which gives us imediately the distributional limit of the regularized solution of the third equation of system (23), i.e.,

$$
N_{\epsilon}^{x}(u) \rightarrow \frac{1}{2} a f^{\prime}\left(x_{0}\right)\left(u_{+}+\theta(u)\right) .
$$

Now we face the most complicated equation of system (23) which contains the distributionally ill-defined expressions, namely

$$
\begin{aligned}
\ddot{N}_{\epsilon}^{v}(u) & =2\left[N_{\epsilon}^{x}(u) f^{\prime}\left(x_{\epsilon}(u)\right) \rho_{\epsilon}(u)\right]^{\cdot}+\left[N_{\epsilon}^{u}(u) f\left(x_{\epsilon}(u)\right) \rho_{\epsilon}(u)\right]^{*} \\
& -N_{\epsilon}^{u}(u) f^{\prime \prime}\left(x_{\epsilon}(u)\right)\left[\theta * \ddot{x}_{\epsilon}\right]^{2}(u) \rho_{\epsilon}(u)-N_{\epsilon}^{x}(u) f^{\prime}\left(x_{\epsilon}(u)\right) \dot{\rho}_{\epsilon}(u) \\
& -N_{\epsilon}^{u}(u) f^{\prime}\left(x_{\epsilon}(u)\right) \ddot{x}_{\epsilon}(u) \rho_{\epsilon}(u),
\end{aligned}
$$

where we have inserted all the dependences explicitly. Note that in addition to the obvious troublesome fifth term which involves the "square" of the $\delta$-distribution $\left(\ddot{x}_{\epsilon} \propto \rho_{\epsilon} !\right)$, also the fourth term contains divergent and regularization-dependent contributions, since the derivatives of $f(x)$ and $N_{\epsilon}^{x}$ produce $\delta$ - and step functions respectively. We are going to calculate the distributional limits of the right hand side of equation (36) term by term, which can easily be done for the first three ones using yet well known techniques.

First, second and third term:

$$
\begin{aligned}
\lim _{\epsilon \rightarrow 0} 2\left[N_{\epsilon}^{x}(u) f^{\prime}\left(x_{\epsilon}(u)\right) \rho_{\epsilon}(u)\right]^{\cdot} & =\frac{1}{2} a f^{\prime}\left(x_{0}\right)^{2} \dot{\delta}, \\
\lim _{\epsilon \rightarrow 0}\left[N_{\epsilon}^{u}(u) f\left(x_{\epsilon}(u)\right) \rho_{\epsilon}(u)\right]^{*} & =a f\left(x_{0}\right) \ddot{\delta}, \\
\lim _{\epsilon \rightarrow 0} N_{\epsilon}^{u}(u) f^{\prime \prime}\left(x_{\epsilon}(u)\right)\left[\theta * \ddot{x}_{\epsilon}\right]^{2}(u) \rho_{\epsilon}(u) & =\frac{1}{12} a f^{\prime}\left(x_{0}\right)^{2} f^{\prime \prime}\left(x_{0}\right) \delta
\end{aligned}
$$

We now come to the expressions involving the divergent and regularizationdependent factors. We outline the calculations in some detail.

Fourth term: $(i v):=-N_{\epsilon}^{x}(u) f^{\prime}\left(x_{\epsilon}(u)\right) \dot{\rho}_{\epsilon}(u)$

By integration by parts we obtain ( $\varphi$ again a test function)

$$
\begin{aligned}
& -\int_{-\epsilon}^{\epsilon} \varphi(u) N_{\epsilon}^{x}(u) f^{\prime}\left(x_{\epsilon}(u)\right) \dot{\rho}_{\epsilon}(u) d u=\underbrace{\int_{-1}^{1} \dot{\varphi}(\epsilon u) N_{\epsilon}^{x}(\epsilon u) f^{\prime}\left(x_{\epsilon}(\epsilon u)\right) \rho(u) d u}_{A} \\
& +\underbrace{\int_{-1}^{1} \varphi(\epsilon u) N_{\epsilon}^{x}(\epsilon u) f^{\prime \prime}\left(x_{\epsilon}(\epsilon u)\right) \dot{x}_{\epsilon}(\epsilon u) \rho(u) d u}_{B}+\underbrace{\int_{-1}^{1} \varphi(\epsilon u) \dot{N}_{\epsilon}^{x}(\epsilon u) f^{\prime}\left(x_{\epsilon}(\epsilon u)\right) \rho(u) d u}_{C} .
\end{aligned}
$$

Inserting $\theta * \theta * \ddot{N}_{\epsilon}^{x}$ according to equations (26) and (27) for $N_{\epsilon}^{x}$ we find for the expressions labelled $A$ and $B$ 


$$
\begin{aligned}
& A \rightarrow-\frac{1}{4} a f^{\prime}\left(x_{0}\right)^{2} \dot{\delta}, \\
& B \rightarrow \frac{1}{12} a f^{\prime}\left(x_{0}\right)^{2} f^{\prime \prime}\left(x_{0}\right) \delta .
\end{aligned}
$$

Finally we have for the most complicated expression

$$
\begin{aligned}
C= & \underbrace{\frac{1}{2 \epsilon} a \int_{-1}^{1} \varphi(\epsilon u) f^{\prime}\left(x_{\epsilon}(\epsilon u)\right) \rho(u) \int_{-1}^{u} f^{\prime}\left(x_{\epsilon}(\epsilon s)\right) \dot{\rho}(s) d s d u}_{C 1} \\
& +\underbrace{\frac{1}{2} a \int_{-1}^{1} \varphi(\epsilon u) f^{\prime}\left(x_{\epsilon}(\epsilon u)\right) \rho(u) \int_{-1}^{u} f^{\prime}\left(x_{\epsilon}(\epsilon s)\right) s \dot{\rho}(s) d s d u}_{C 2} \\
& +\underbrace{\int_{-1}^{1} \varphi(\epsilon u) f^{\prime}\left(x_{\epsilon}(\epsilon u)\right) \rho(u) \int_{-1}^{u}\left[a f^{\prime}\left(x_{\epsilon}(\epsilon s)\right)+\frac{1}{2} N_{\epsilon}^{x}(\epsilon s) f^{\prime \prime}\left(x_{\epsilon}(\epsilon s)\right)\right] \rho(s) d s d u}_{C 3} .
\end{aligned}
$$

The term labeled $C 1$ diverges due to the factor $\epsilon^{-1}$. However, as we shall see later, this expression combined with another one arising from the fifth term of equation (36) will provide us with a finite result.

The term labelled $C 2$ involves a regularization-dependent factor, i.e.,

$$
C 2 \rightarrow \frac{1}{2} a f^{\prime}\left(x_{0}\right)^{2} \delta \int_{-1}^{1} \rho(u) \int_{-1}^{u} s \dot{\rho}(s) d s d t .
$$

As in the case of the previous term $C 1$, this problem will be resolved later on, so that we are now left with the task of computing the limit of $C 3$. Again using similar techniques we obtain

$$
C 3 \rightarrow \frac{1}{2} a f^{\prime}\left(x_{0}\right)^{2} \delta+\frac{1}{24} a f^{\prime}\left(x_{0}\right)^{2} f^{\prime \prime}\left(x_{0}\right) \delta .
$$

Collecting together the results of equations (39), (40) and (43), we finally have

$$
(i v)-C 1-C 2 \rightarrow-\frac{1}{4} a f^{\prime}\left(x_{0}\right)^{2} \dot{\delta}+\frac{1}{8} a f^{\prime}\left(x_{0}\right)^{2} f^{\prime \prime}\left(x_{0}\right) \delta+\frac{1}{2} a f^{\prime}\left(x_{0}\right)^{2} \delta,
$$

where now, by an abuse of notation $C 1$ and $C 2$ denote the respective quantities in equation (41) without integration over the test function.

Now we finally come to the last term in equation (36) which will resolve the problems with the expressions $C 1$ and $C 2$.

Fifth term: $(v):=-N_{\epsilon}^{u}(u) f^{\prime}\left(x_{\epsilon}(u)\right) \ddot{x}_{\epsilon}(u) \rho_{\epsilon}(u)$

Inserting solution (25) we find

$$
\begin{aligned}
& -\int_{-\epsilon}^{\epsilon} \varphi(u) N_{\epsilon}^{u}(u) f^{\prime}\left(x_{\epsilon}(u)\right) \ddot{x}_{\epsilon}(u) \rho_{\epsilon}(u) d u \\
& =\underbrace{-\frac{1}{2 \epsilon} a \int_{-1}^{1} \varphi(\epsilon u) f^{\prime}\left(x_{\epsilon}(\epsilon u)\right)^{2} \rho(u)^{2} d u}_{(*)} \underbrace{-\frac{1}{2} a \int_{-1}^{1} \varphi(\epsilon u) f^{\prime}\left(x_{\epsilon}(\epsilon u)\right)^{2} u \rho(u)^{2} d u}_{(* *)} .
\end{aligned}
$$


We see now that the expression $(*)$ diverges, but that the sum $C 1+(*)$ converges. More precisely, by integration by parts we get

$$
\begin{aligned}
C 1+(*) & =-\frac{1}{4} a \int_{-1}^{1} \varphi(\epsilon u) f^{\prime}\left(x_{\epsilon}(\epsilon u)\right) \rho(u) \int_{-1}^{u} f^{\prime \prime}\left(x_{\epsilon}(\epsilon s)\right) \rho(s) \int_{-1}^{s} f^{\prime}\left(x_{\epsilon}(\epsilon r)\right) \rho(r) d r d s d u \\
& \rightarrow-\frac{1}{4} a \varphi(0) f^{\prime}\left(x_{0}\right)^{2} f^{\prime \prime}\left(x_{0}\right) \underbrace{\int_{-1}^{1} \rho(u) \int_{-1}^{u} \rho(s) \int_{-1}^{s} \rho(r) d r d s d u}_{1 / 6} \\
& =-\frac{1}{24} a \varphi(0) f^{\prime}\left(x_{0}\right)^{2} f^{\prime \prime}\left(x_{0}\right) .
\end{aligned}
$$

Hence we have the distributional limit

$$
C 1+(*) \rightarrow-\frac{1}{24} a f^{\prime}\left(x_{0}\right)^{2} f^{\prime \prime}\left(x_{0}\right) \delta .
$$

On the other hand expression $(* *)$ contains a regularization-dependent factor which is proportional to the one contained in the term $C 2$. More precisely, from equation (42) and the fact that

$$
(* *) \rightarrow-\frac{1}{2} a f^{\prime}\left(x_{0}\right)^{2} \delta \int_{-1}^{1} u \rho(u)^{2} d u
$$

we have

$$
\begin{aligned}
C 2+(* *) & \rightarrow \frac{1}{2} a f^{\prime}\left(x_{0}\right)^{2} \delta \underbrace{\left[\int_{-1}^{1} \rho(u)\left[\int_{-1}^{u} s \dot{\rho}(s) d s-u \rho(u)\right] d u\right]}_{1 / 2} \\
& =-\frac{1}{4} a f^{\prime}\left(x_{0}\right)^{2} \delta .
\end{aligned}
$$

We are now ready to collect together all five terms of equation (36), i.e., of the second equation of the regularized system (23). From equations (37), (44), (47) and (49) we get the following result:

$$
\ddot{N}_{\epsilon}^{v} \rightarrow a f\left(x_{0}\right) \ddot{\delta}+\frac{1}{4} a f^{\prime}\left(x_{0}\right)^{2}(\dot{\delta}+\delta),
$$

which, using initial condition (24), we readily integrate to

$$
N_{\epsilon}^{v}(u) \rightarrow b(1+u)+a\left[f\left(x_{0}\right) \delta+\frac{1}{4} f^{\prime}\left(x_{0}\right)^{2}\left(\theta(u)+u_{+}\right)\right] .
$$

Finally we have for the whole system (23) the following (distributional limits of the) solutions

$$
\begin{aligned}
& N_{\epsilon}^{u}(u)=a(1+u) \\
& N_{\epsilon}^{v}(u) \rightarrow b(1+u)+a\left[f\left(x_{0}\right) \delta+\frac{1}{4} f^{\prime}\left(x_{0}\right)^{2}\left(\theta(u)+u_{+}\right)\right] \\
& N_{\epsilon}^{x}(u) \rightarrow \frac{1}{2} a f^{\prime}\left(x_{0}\right)\left(\theta(u)+u_{+}\right) \\
& N_{\epsilon}^{y}(u)=0
\end{aligned}
$$


Hence, viewed distributionally, the Jacobi field suffers a kink, a jump and a $\delta$-like pulse in the $v$-direction as well as a kink and jump in the $x$-direction overlapping the linear flat space behavior. These effects can be understood heuristically from the corresponding behavior of the geodesics, given by equation (18). The constant factor $a$, which gives the "scale" of all the nonlinear effects, arises from the "time advance" of the "nearby" geodesics, represented by the initial velocity of the Jacobi field in the $u$-direction (cf. initial conditions (24)).

Note, however, that this "time advance" is not the only effect generically generating deviations from the flat space behavior, but rather arises as an artifact of our initial conditions. One can easily show that different initial conditions on the deviation vector field, even without "time advance", produce kinks and jumps as well. For example, let

$$
N_{\epsilon}^{a}(-1)=(0,0,0,0) \text { and } \dot{N}_{\epsilon}^{a}(-1)=(0, a, b, 0) ;
$$

then a similar (but now even simpler) calculation leads to the following (distributional limits of the) regularized Jacobi field

$$
\begin{aligned}
& N_{\epsilon}^{u}=0, \\
& N_{\epsilon}^{v} \rightarrow a(1+u)+\frac{1}{4} b f^{\prime}\left(x_{0}\right) f^{\prime \prime}\left(x_{0}\right) u_{+}+b f^{\prime}\left(x_{0}\right) \theta(u), \\
& N_{\epsilon}^{x} \rightarrow b(1+u)+\frac{1}{2} b f^{\prime \prime}\left(x_{0}\right) u_{+}, \\
& N_{\epsilon}^{y}=0 .
\end{aligned}
$$

The kink of the $x$-component of the deviation field now arises from the fact that a "nearby" geodesic passes the shock at an $x$-value of $x_{0}+b$, hence according to equation (18) suffers a kink of "strength" $(1 / 2) f^{\prime}\left(x_{0}+b\right)$. Taylor expansion yields $f^{\prime}\left(x_{0}+b\right) \approx f^{\prime}\left(x_{0}\right)+f^{\prime \prime}\left(x_{0}\right) b$, such that the kink difference of "nearby" geodesics is given by $(1 / 2) f^{\prime \prime}\left(x_{0}\right) b$, which is exactly the factor given in the third equation of (54). The kink and jump in $v$-direction can be explained by similar heuristic arguments.

\section{CONCLUSION}

We have shown that the geodesic and Jacobi equation of impulsive pp-waves can be treated consistently using the distributional form of the space time metric (2). The main problem was to deal with products ill-defined within Schwartz's linear distribution theory. The geodesic equation involves terms proportional to " $\theta \delta$ " while the Jacobi equation, although linear in the components of the deviation vector field, contains even more singular terms like " $\delta^{2}$ " and " $\theta^{2} \delta$ ". Our strategy consisted essentially in a careful and general regularization procedure, replacing the $\delta$-distribution by a mollifying sequence $\rho_{\epsilon}$. While mathematically sound, this procedure corresponds to the physical idea of viewing the impulsive wave as the limiting case of a sandwich wave of ever decreasing support but constant strength. Our approach leads to smooth solutions of the regularized equations which possess a regularization-independent limit within the vector space of distributions. However, this is not obvious (cf. for example11), but rather should strengthen our trust in impulsive waves as reasonable solutions of Einstein's equations. Furthermore our distributional "solutions" of the geodesic and Jacobi equation perfectly coincide with physical expectations, showing that the geometry of impulsive pp-waves can be described in a consistent way using the distributional form of the metric, as 
long as one applies proper regularization methods as opposed to "ad-hoc strategies" involving certain "multiplication rules". In fact when dealing with nonlinear operations (of a certain complexity) on singular, i.e., distributional data, reliable results can only be achieved by careful regularization procedures (cf. the comments on "common errors" even in the mathematical literature given by Hájek 12 ).

However from an even more formal point of view, the approach taken in this paper suffers from the fact that we do not really have an elaborated solution concept for the regularized equations Such a notion is provided by the theory of Nonlinear Generalized Functions 19.13.14 due to J. F. Colombeau and others, where - loosely speaking - the regularized sequence is viewed as one mathematical object, i.e., a generalized function. Recently, singular, nonlinear ODEs (exactly the type of equations we were dealing with) have been studied in the context of this new mathematical framework 4,6 , providing a powerful tool to handle both, singular data and especially this type of nonlinear differential equations. Future work will be concerned with the analysis of the geodesic and Jacobi equation for pp-waves from the point of view of this new solution concept.

\section{Acknowledgement}

The author owes special thanks to M. Kunzinger for great support with the calculations and M. Oberguggenberger and M. Grosser for many helpful discussions. This work was supported by Austrian Academy of Science, Ph.D. programme, Grant \#338.

\section{APPENDIX:}

In this appendix we prove (for small $\epsilon$ ) the global existence and uniform boundedness (in $\epsilon$ ) of the solutions to the last equation of system (7). For simplicity we only treat the one-dimensional case and write $g$ instead of $D f$. The main idea is to use \|\|$_{1^{-}}$ bounds on the regularizing sequence $\rho_{\epsilon}$. We start with the following statement.

Proposition: Let $g: \mathbb{R} \rightarrow \mathbb{R}$ smooth and $\rho \in \mathcal{D}([-1,1]), \int \rho=1, \rho_{\epsilon}(x):=$ $(1 / \epsilon) \rho(x / \epsilon)$. For fixed $\epsilon$ consider the differential equation

$$
\ddot{x}_{\epsilon}(t)=g\left(x_{\epsilon}(t)\right) \rho_{\epsilon}(t),
$$

with initial conditions $x_{\epsilon}(-1)=x_{0}$ and $\dot{x}_{\epsilon}(-1)=\dot{x}_{0}>0$. Then there exists a unique solution $x_{\epsilon}(t)$ on the interval $J_{\epsilon}:=[-1,-\epsilon+\alpha]$, where

$$
\alpha:=\min \left\{\frac{b}{\|g\|_{\infty, I}\|\rho\|_{1}+\dot{x}_{0}}, \frac{1}{2 L}\right\} \quad(b>0),
$$

$I:=\left\{x \in \mathbb{R}:\left|x-x_{0}\right| \leq b+\dot{x}_{0}\right\}$ and $L$ is a Lipschitz constant for $g$ on $I$.

The Proof is just a modification of the proof of the classical (first order) ODE existence and uniqueness theorem. We work on the (nonempty, closed) subset $X_{\epsilon}:=$ $\left\{x_{\epsilon} \in C\left(J_{\epsilon}\right):\left|x_{\epsilon}(t)-x_{0}\right| \leq b+\dot{x}_{0}\right\}$ of the Banach space of continuous functions on the interval $J_{\epsilon}$. Since no first order derivative of $x_{\epsilon}$ enters the right hand side of equation (A1) we may define the integration operator $A$ by

$$
\left[A x_{\epsilon}\right](t):=x_{0}+\dot{x}_{0}(t+1)+\int_{-\epsilon}^{t} \int_{-\epsilon}^{s} g\left(x_{\epsilon}(r)\right) \rho_{\epsilon}(r) d r d s
$$


By construction $A x_{\epsilon} \in C\left(J_{\epsilon}\right)$, but we even have $A x_{\epsilon} \in X_{\epsilon}$ since

$$
\begin{aligned}
\left|\left[A x_{\epsilon}\right](t)-x_{0}\right| & \leq \dot{x}_{0}(t+1)+\int_{-\epsilon}^{t} \int_{-\epsilon}^{s}\left|g\left(x_{\epsilon}(r)\right)\right|\left|\rho_{\epsilon}(r)\right| d r d s \\
& \leq \alpha\left(\dot{x}_{0}+\|g\|_{\infty, I}\|\rho\|_{1}\right)+\dot{x}_{0} \leq b+\dot{x}_{0}
\end{aligned}
$$

By a similar estimate one shows that $A$, in fact, is a contraction on $X_{\epsilon}$. Hence by the fixed point theorem we have a unique solution $x_{\epsilon} \in X_{\epsilon}$.

Since $\alpha$ is independent of $\epsilon$ we have for small $\epsilon$ : $-\epsilon+\alpha \geq \epsilon$. But at $t=\epsilon$ the right hand side of the differential equation (A1) has already been "turned off". Hence for small $\epsilon$ the solutions are defined on the whole real line.

Furthermore, since for all $\epsilon$ the solution lies in $X_{\epsilon}, x_{\epsilon}$ is bounded uniformly in $\epsilon$ for say $t \leq \alpha / 2$ (for $\epsilon$ small enough). For larger values of $t x_{\epsilon}$ grows only linearly with $\dot{x}_{\epsilon}$ which is bounded by $\dot{x}_{0}+\|g\|_{\infty, I}\|\rho\|_{1}$. Hence (for small $\epsilon$ ) $x_{\epsilon}(t)$ is bounded uniformly in $\epsilon$ on every compact set.

${ }^{1}$ J. Ehlers, W. Kundt, "Exact solutions of the gravitational field equations," in Gravitation, An Introduction to Current Research, edited by L. Witten (Wiley, New York, 1964) pp. 49-99.

${ }^{2}$ R. Penrose, "The geometry of impulsive gravitational waves," in General Relativity, Papers in Honour of J. L. Synge, edited by L. O'Raifeartaigh (Clarendon Press, Oxford, 1972), pp. 101-115.

${ }^{3}$ P. C. Aichelburg, R. U. Sexl, "On the gravitational field of a massless particle," J. Gen. Rel. Grav. 2, 303-312 (1971).

${ }^{4}$ H. Balasin, H. Nachbagauer, "The ultrarelativistic Kerr-geometry and its energymomentum tensor," Class. Quantum Grav. 12, 707-713 (1995).

${ }^{5}$ C. O. Loustó, N. Sánchez, "The ultrarelativistic limit of the Kerr-Newman geometry and particle scattering at the Planck scale," Phys. Lett. B 232, 462-466 (1989).

${ }^{6}$ R. Penrose, "Structure of space-time," in Battelle Recontres edited by C. M. DeWitt and J. A. Wheeler (Benjamin, New York, 1968), pp. 121-235.

7 T. Dray, G. 't Hooft, "The gravitational shockwave of a massless particle," Nucl. Phys. B 232, 173-188 (1985).

${ }^{8}$ V. Ferrari, P. Pendenza, G. Veneziano, "Beam like gravitational waves and their geodesics," J. Gen. Rel. Grav. 20 (11), 1185-1191 (1988).

${ }^{9}$ H. Balasin, "Geodesics for impulsive gravitational waves and the multiplication of distributions," Class. Quantum Grav. 14, 455-462 (1997).

${ }^{10}$ M. Oberguggenberger, Multiplication of Distributions and Applications to Partial Differential Equations, (Longman Scientific and Technical, New York, 1992).

${ }^{11}$ R. Steinbauer, "The ultrareltivistic Reissner-Nordstrøm field in the Colombeau algebra," J. Math. Phys. 38 (3), 1614-1622 (1997).

12 O. Hájek, Bull. Am. Math. Soc. 12 (2), 272-279, 1985.

13 J. F. Colombeau, New Generalized Functions and Multiplication of Distributions, (North Holland, Amsterdam, 1984).

14 J. F. Colombeau, Multiplication of Distributions, (Springer, Berlin, 1992).

${ }^{15}$ R. Hermann, M. Oberguggenberger, "Generalized functions, calculus of variations and nonlinear ODEs," preprint (1995).

${ }^{16}$ M. Kunzinger, "Lie Transformation Groups in Colombeau Algebras," Ph.D. thesis, University of Vienna 1996. 